\title{
Recurrent septic arthritis due to Pseudomonas sp.
}

\author{
Michael DAN* \\ M.D.
}

\author{
Medina JeDWAB \\ M.D.
}

\author{
Shlomo ShiBolet \\ M.D.
}

Department of Medicine D, Ichilov Hospital, Tel-Aviv, Israel

\begin{abstract}
Summary
Two episodes of Pseudomonas septic arthritis occurred in an 84-year-old diabetic man separated by a 6 year interval. Pseudomonas is an unusual cause of septic arthritis requiring vigorous antibiotic therapy. The recurrence of the infection may be related to the inadequate response to treatment in the first episode, however, the long interval remains unexplained.
\end{abstract}

\section{Introduction}

Although the occurrence of generalized Gramnegative infections have increased, joint infections due to these organisms are still infrequent. Pseudomonas aeruginosa is an uncommon cause, being found in only $2 \%$ of patients with septic arthritis (Sharp et al., 1979). The majority of recently reported Pseudomonas arthritis cases occurred in drug addicts (Tindel and Crowder, 1971; Gifford et al., 1975). The purpose of this report is to describe an unusual case of recurrent Pseudomonas arthritis in an elderly non-addict patient.

\section{Case report}

An 84-year-old man was admitted with a 1-day history of pain in the right knee and fever. The patient had had diabetes mellitus and hypertension for many years. There was a 15-year history of gonarthrosis in both knees. Six years before, the patient had been admitted for swelling and pain of the same knee with fever which appeared 2 weeks following an intra-articular injection of hydrocortisone. Needle aspiration of the joint purulent fluid, and $\boldsymbol{P}$. aeruginosa was grown on a culture. Gentamicin sulphate $240 \mathrm{mg}$ a day, was given for 20 days. On the 16th hospital day Pseudomonas was again recovered on culture from the same

\footnotetext{
*Present address: Division of Infectious Diseases, Department of Medicine, Clinical Science Building, The University of Alberta, Edmonton, Alberta T6G 2G3, Canada.
}

knee. When the patient was readmitted one month later for plaster cast removal, the knee was still swollen and hot and the patient was febrile. Administration of gentamicin sulphate $160 \mathrm{mg}$ a day for 10 days, was followed by regression of symptoms.

During the following years the patient continued to experience knee pains from time to time, but no signs of acute arthritis were noted. No additional intra-articular injection was performed.

On the present admission blood pressure was $160 / 80 \mathrm{mmHg}$; pulse rate $90 / \mathrm{min}$ and regular; and temperature $38^{\circ} \mathrm{C}$. The right knee was swollen, tender and warm. The findings from the remainder of the examination were non-contributory.

Haemoglobin level was $14.3 \mathrm{~g} / \mathrm{dl}$, and white blood cell count was $10.4 \times 10^{9} / 1$ with marked left shift. Erythrocyte sedimentation rate was $50 \mathrm{~mm} / \mathrm{hr}$, and glucose was $140 \mathrm{mg} / 100 \mathrm{ml}$. Blood urea nitrogen and results of urine analysis were normal. Blood and urine cultures were sterile. X-ray of the right knee showed severe degenerative changes. Five millilitres of purulent fluid, containing $75.0 \times 10^{9}$ leucocytes $/ 1$ with $91 \%$ neutrophils, was removed. Culture of this fluid grew $\boldsymbol{P}$. aeruginosa. Gentamicin sulphate, $240 \mathrm{mg}$ a day, and carbenicillin, $24 \mathrm{~g}$ a day, was administered intravenously for 2 weeks. A plaster cast was applied because of severe pains. The temperature went down rapidly and the local signs regressed as well. Surgical intervention aimed to local exploration and arthrodesis was refused by the patient. At discharge on the 33rd hospital day, the patient was afebrile and the sedimentation rate was $18 \mathrm{~mm} / \mathrm{hr}$.

\section{Discussion}

Certain host factors appear to predispose patients to the development of septic arthritis as well as alter the course of these infections (Goldenberg and Cohen, 1976). These factors are more prominent in Gramnegative arthritis explaining the poor therapeutic 
results achieved in this group (Goldenberg et al., 1974).

The predisposing factors are: concurrent extraarticular infection; underlying chronic disease; previous antibiotic or immunosuppressive therapy; and previously diseased joint. The reported patient suffered from diabetes mellitus, and gonarthrosis, which was treated with intra-articular injections of corticosteroids.

The source of Pseudomonas in this case was probably the intra-articular injection performed 2 weeks before the first admission.

Combined administration of gentamicin and carbenicillin should be considered in all Pseudomonas joint infections, not only in those in which organisms are shown to be relatively resistant to one or the other drug. The combination of gentamicin and carbenicillin is synergistic against one third of the Pseudomonas isolates (Klastersky, Swings and Daneau, 1970). High concentrations of both the agents can be reached in joint fluids by systemic administration (Chow, Hecht and Winters, 1971). Effective concentrations of carbenicillin require a dose of $20-40 \mathrm{~g}$ per day. This high dosage is of particular importance in infections of bone and soft tissue in which sub-optimum dosage may promote development of resistant Pseudomonas strains. Bactericidal gentamicin concentrations in synovial fluid should be reached by intramuscular or intravenous administration of the usual doses of the drug. The present patient benefited from this powerful antibiotic combination only in the second episode, while gentamicin alone had been administered in the first attack. This inadequate course of antibiotic therapy, together with the several predisposing factors encountered in the reported patient, may explain the delayed recovery in the first episode and the recurrence that followed.
Reviewing the literature about Gram-negative arthritis, one is impressed by the remarkably poom therapeutic results (Sharp et al., 1979; Goldenberg et al., 1974; Goldenberg and Cohen, 1976). However: no similar case of Pseudomonas arthritis recurrence with a 6-year interval, was found in the literatures Only in one case, of a heroin user, was Pseudomona옹 regrown from the previously infected joint after $1 \overline{\mathrm{p}}$ months; but the authors considered this as re $\$$ infection (Gifford et al., 1975).

It remains unclear why the interval between the 2 episodes was so prolonged. A residuaP dormant osteitic focus adjacent to the joint might serve as a source of reinfection. This explanation remains hypothetical as the patient refused surgicas exploration.

\section{References}

Chow, A., Hecht, K. \& Winters, R. (1971) Gentamicin and carbenicillin penetration into the septic joint (letter) New England Journal of Medicine, 285, 178.

Gifford, D.B., Patzakis, M., IVler, D. \& Swezey, R.L음 (1975) Septic arthritis due to Pseudomonas in heroinaddicts. Journal of Bone and Joint Surgery, 57-A, 631.

Goldenberg, D.L., BrandT, K.D., CathCart, E.S. \& CoHen, A.S. (1974) Acute arthritis caused by Gram= negative bacilli: a clinical characterization. Medicinge् Baltimore, 53, 197.

GoldenberG, D.L. \& Cohen, A.S. (1976) Acute infectious arthritis: a review of patients with nongonococcal jengn infection (with emphasis on therapy and prognos s) American Journal of Medicine, 60, 369.

Klastersky, J., Swings, G. \& Daneau, D. (1970) Antī microbial activity of the carbenicillin/gentamicin come bination against Gram-negative bacilli. American Journad of the Medical Sciences, 260, 373.

Sharp, J. T., LiDSky, M.D., DufFY, J. \& Duncan, M.wō (1979) Infectious arthritis. Archives of Internal Medicine? 139, 1125.

Tindel, J.R. \& Crowder, J.G. (1971) Septic arthritis due to Pseudomonas aeruginosa. Journal of the American Medicati Association, 218, 554. 